\title{
Particle production and flow-like effects in small systems
}

\author{
Antonio Ortiz, for the ALICE, ATLAS, CMS and LHCb Collaborations* \\ Instituto de Ciencias Nucleares, UNAM, Circuito Exterior s/n, CU, Deleg. Coyoacán, Ciudad de \\ México 04510 \\ E-mail: antonio.ortiz@nucleares.unam.mx
}

Particle production in small systems ( $\mathrm{pp}$ and $\mathrm{p}-\mathrm{Pb}$ collisions) has unveiled unexpected collectivelike behavior. In this work an overview of the current investigation on the similarities between small systems and heavy-ion collisions is presented. Recent results from the experiments at the LHC are discussed. They include measurements of multi-particle correlations, as well as, identified particle production as a function of charged-particle multiplicity density, and more recently, as a function of transverse spherocity.

7th Annual Conference on Large Hadron Collider Physics - LHCP2019

20-25 May, 2019

Puebla, Mexico

${ }^{*}$ Speaker. 


\section{Introduction}

The goal of the heavy-ion program is to understand the behavior of Quantum Chromo-Dynamics (QCD) at high temperatures and densities. Results at the Large Hadron Collider (LHC) confirmed the formation of a new form of matter characterized by deconfinement, which is compatible with the theoretically predicted Quark-Gluon Plasma (QGP) [1]. It has been found that the low transverse momentum $\left(p_{\mathrm{T}}<2 \mathrm{GeV} / c\right)$ hadron production and multi-particle correlation measurements are well-described by fluid dynamic models, yielding that matter properties are consistent with a perfect fluid that exhibits small shear viscosity over entropy ratio, $\eta / s[2]$. Other observables such as jet suppression are also well-described by models which assume the formation of a QGP [3]. The main conclusions arose from comparisons of heavy-ion data with reference data, such as minimumbias pp and $\mathrm{p}-\mathrm{A}$ collisions, where no signatures of jet quenching were observed.

The large amount of LHC pp data, allowed two-particle azimuthal correlations to be measured as a function of the charged-particle multiplicity $\left(N_{\mathrm{ch}}\right)$. For the first time, very similar azimuthal anisotropies as in heavy-ion collisions were observed in high-multiplicity pp collisions at $\sqrt{s}=$ $7 \mathrm{TeV}$ [4]. The analysis was further extended to lower and higher energies [5], as well as other systems such as $\mathrm{p}-\mathrm{Pb}$ at $\sqrt{s_{\mathrm{NN}}}=5.02 \mathrm{TeV}[6,7]$. Figure 1 shows the correlation for particles with $1<p_{\mathrm{T}}<2 \mathrm{GeV} / c$ in low and very-high multiplicity $\mathrm{p}-\mathrm{Pb}$ events. The long-range structure (often referred to as the near-side ridge) centred at $\Delta \phi=0$ and elongated over the full measured $\Delta \eta$ range of 2.9 units is not present in the corresponding low-activity sample. Moreover, also for the first time, two long-range ridge-like structures, one on the near side and one on the away side, were observed when the per-trigger yield obtained in low-multiplicity events was subtracted from the one in high-multiplicity events. This suggested that the near-side ridge is accompanied by an essentially identical away-side ridge.
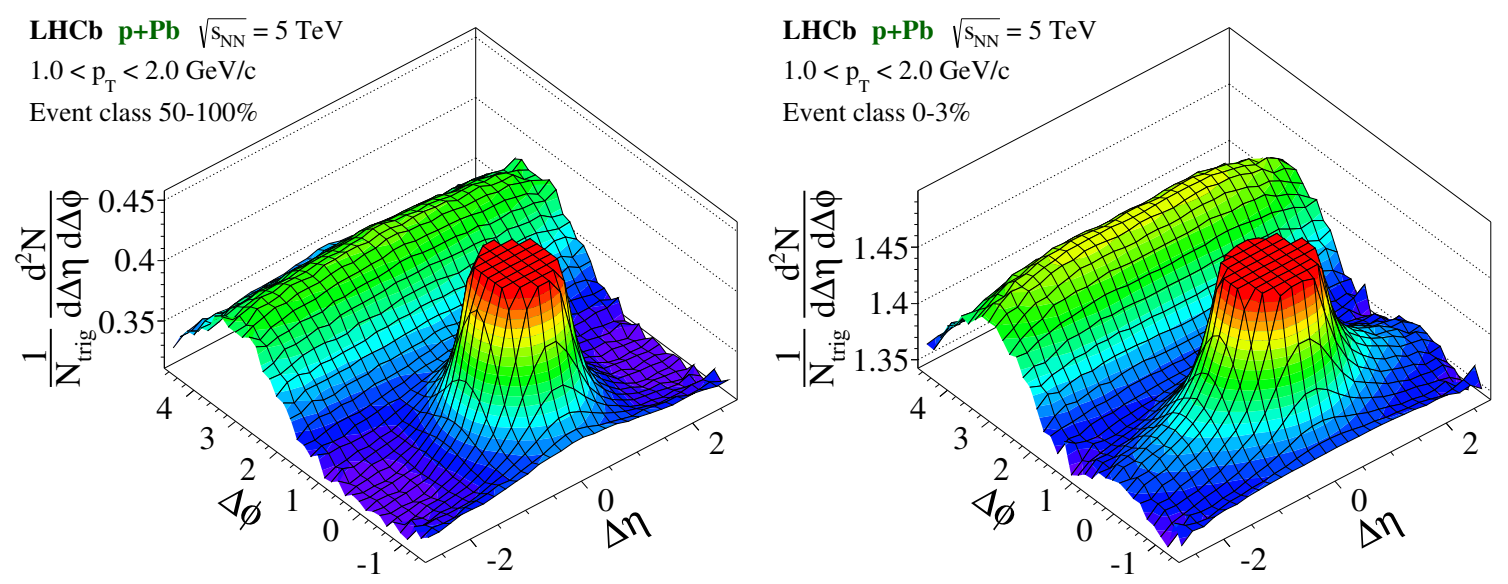

Figure 1: Two-particle correlation for (left) low- and (right) high-event activity in p-Pb collisions at $\sqrt{s_{\mathrm{NN}}}=$ $5.02 \mathrm{TeV}$ measured with the LHCb detector [7]. Primary charged particles were selected in a $p_{\mathrm{T}}$ range of $1-2 \mathrm{GeV} / c$. The near-side peak around $\Delta \eta=\Delta \phi=0$ is truncated in the histograms.

Naturally, it was suggested that these anisotropies in small systems could have the same origin as in heavy-ion collisions, namely the hydrodynamic response of the produced medium to the initial shape of the interaction region in the transverse plane [8]. However, the main concern was on the 
applicability of hydrodynamics to small non-equilibrium systems. A big progress in this direction has been accomplished, for example using numerical holography, it has been demonstrated that hydrodynamics can describe the evolution of systems as small as $R \sim 1 / T_{\text {eff }}, T_{\text {eff }}$ being the effective temperature [9]. From the initial state perspective, the azimuthal anisotropy is due to initial state correlations present in the nuclear wave functions of the incoming nuclei [10]. The main question is whether azimuthal anisotropies established during the initial stages of the collision can survive subsequent final state interactions [11]. The third approach relies on partonic and hadronic transport models, for example AMPT [12]. This model qualitatively (and sometimes quantitatively) describes small system flow signals for various collision systems and energies. The big issue is that in contrast to fluid dynamic simulation, its applicability relies on a sufficiently large mean free path, which is hard to reconcile with the idea of the strongly coupled hydrodynamic system.

From the experimental side, one challenge for small collision systems is the strong correlation between multiplicity (sensitive to low- $p_{\mathrm{T}}$ particles) and hard physics (high- $p_{\mathrm{T}}$ particles) [13]. It has been shown to be reduced if event multiplicity is determined in a pseudorapidity region far from that where the observable of interest is measured. However, an additional treatment of the unwanted particle correlations (from jets or resonances) has to be implemented in data analysis.

It is clear that we are still far from fully understanding the similarities between small systems and heavy-ion data. A more detailed review on small systems can be found in Ref. [14]. In this proceedings, a discussion of recent results on identified particle production and multi-particle correlations in small systems is presented in Sections 2 and 3, respectively. Finally, Section 4 presents a summary of the main results.

\section{Identified particle production as a function of multiplicity}

For pp collisions at $\sqrt{s}=7 \mathrm{TeV}$, the $p_{\mathrm{T}}$ spectra of different particle species $\left(\pi^{ \pm}, \mathrm{K}^{ \pm}, \mathrm{K}_{\mathrm{S}}^{0}\right.$, $\mathrm{K}^{*}(892)^{0}, \mathrm{p}, \overline{\mathrm{p}}, \phi(1020), \Lambda, \bar{\Lambda}, \Xi^{-}, \bar{\Xi}^{+}, \Omega^{-}$and $\bar{\Omega}^{+}$) have been measured as a function of the event multiplicity and over a broad $p_{\mathrm{T}}$ range [15]. In particular, at low $p_{\mathrm{T}}\left(p_{\mathrm{T}}<3 \mathrm{GeV} / c\right)$, all spectra are observed to become harder with increasing charged-particle multiplicity density, the effect is more pronounced for heavier particles. Moreover, the modification of $p_{\mathrm{T}}$ spectra with respect to the inclusive measurement follows a different pattern for mesons and baryons, except for resonances, which follow baryons at a low- $p_{\mathrm{T}}$ of up to approximately $2 \mathrm{GeV} / c$ and tend to be modified similarly to mesons above a $p_{\mathrm{T}}$ of $2 \mathrm{GeV} / c$. Furthermore, it was demonstrated that the evolution of the baryon/meson ratios as a function of $\left\langle\mathrm{d} N_{\mathrm{ch}} / \mathrm{d} \eta\right\rangle$ exhibits an universal pattern for all collision systems [16]. This behavior might indicate a common mechanism at work that depends solely on final-state multiplicity density.

Figure 2 shows the ratios of the yields of $\mathrm{K}_{\mathrm{S}}^{0}, \Lambda, \Xi$ and $\Omega$ to the pion $\left(\pi^{+}+\pi^{-}\right)$yield as a function of $\left\langle\mathrm{d} N_{\mathrm{ch}} / \mathrm{d} \eta\right\rangle$ for $\mathrm{pp}, \mathrm{p}-\mathrm{Pb}$ and $\mathrm{Pb}-\mathrm{Pb}$ collisions at the LHC energies. It is worth noting that the ALICE results for small systems [18] are now compared with the latest data from $\mathrm{Pb}-\mathrm{Pb}$ collisions at $\sqrt{s_{\mathrm{NN}}}=5.02 \mathrm{TeV}$. A significant enhancement of strange to non-strange hadron production is observed with increasing particle multiplicity in pp collisions. The behavior observed in pp collisions resembles that of $\mathrm{p}-\mathrm{Pb}$ collisions at a slightly lower centre-of-mass energy, in terms of both the values of the ratios and their evolution with multiplicity. Strangeness production in heavy-ion collisions can be described in the framework of statistical-hadronisation models [19, 20]. While in 


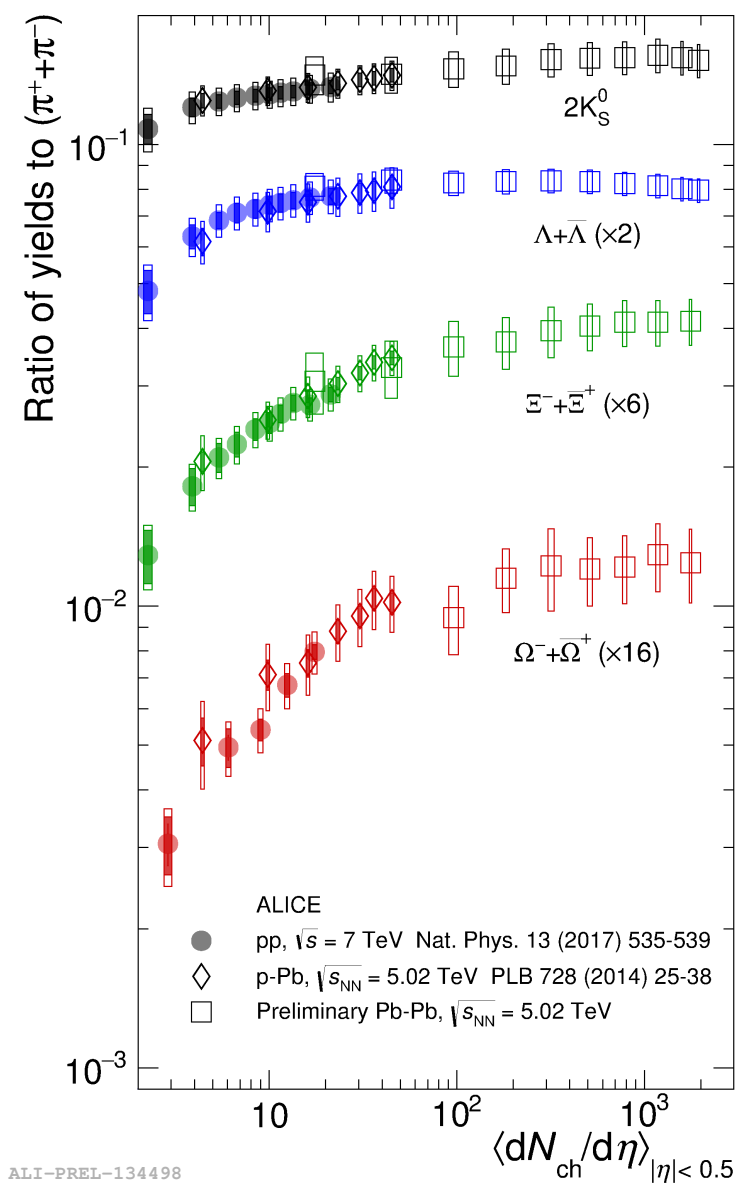

Figure 2: $p_{\mathrm{T}}$-integrated yield ratios to pions $\left(\pi^{+}+\pi^{-}\right)$as a function of $\left\langle\mathrm{d} N_{\mathrm{ch}} / \mathrm{d} \eta\right\rangle$ measured in $|y|<0.5$. The error bars show the statistical uncertainty, whereas the empty and dark-shaded boxes show the total systematic uncertainty and the contribution uncorrelated across multiplicity bins, respectively. The values measured for pp collisions at $\sqrt{s}=7 \mathrm{TeV}$ [15] are compared with results obtained in $\mathrm{p}-\mathrm{Pb}$ [17] and $\mathrm{Pb}-\mathrm{Pb}$ collisions at the LHC.

central heavy-ion collisions, the yields of strange hadrons is consistent with the expectation from a grand-canonical ensemble; the strange hadron yields in elementary collisions, such as pp and $\mathrm{e}^{+}+\mathrm{e}^{-}$, are suppressed with respect to the predictions of the (grand-canonical) thermal models. In the strangeness-canonical approach, within the framework of the THERMUS [21] statistical hadronisation model, the multiplicity and system size dependence of all measured light flavour hadrons can be described, within 2 standard deviations [22].

The ALICE Collaboration has performed the strangeness analysis using pp data at $\sqrt{s}=$ $13 \mathrm{TeV}$ [23], and compared data with MC event generators predictions. PYTHIA 8.210 [24] (tune Monash [25]) gives a reasonable description of the $\mathrm{K}_{\mathrm{S}}^{0}$ results, however it shows a less pronounced increase of the strange baryon yields with increasing $N_{\mathrm{ch}}$ than what is observed in the data. Another generator is DIPSY [26] which incorporates the so-called "rope hadronization". It does a better job in the description of data than PYTHIA, however, the discrepancy for the $\Omega$ distributions remains large. The EPOS-LHC [27] model yields similar conclusions. The results suggest that the origin 


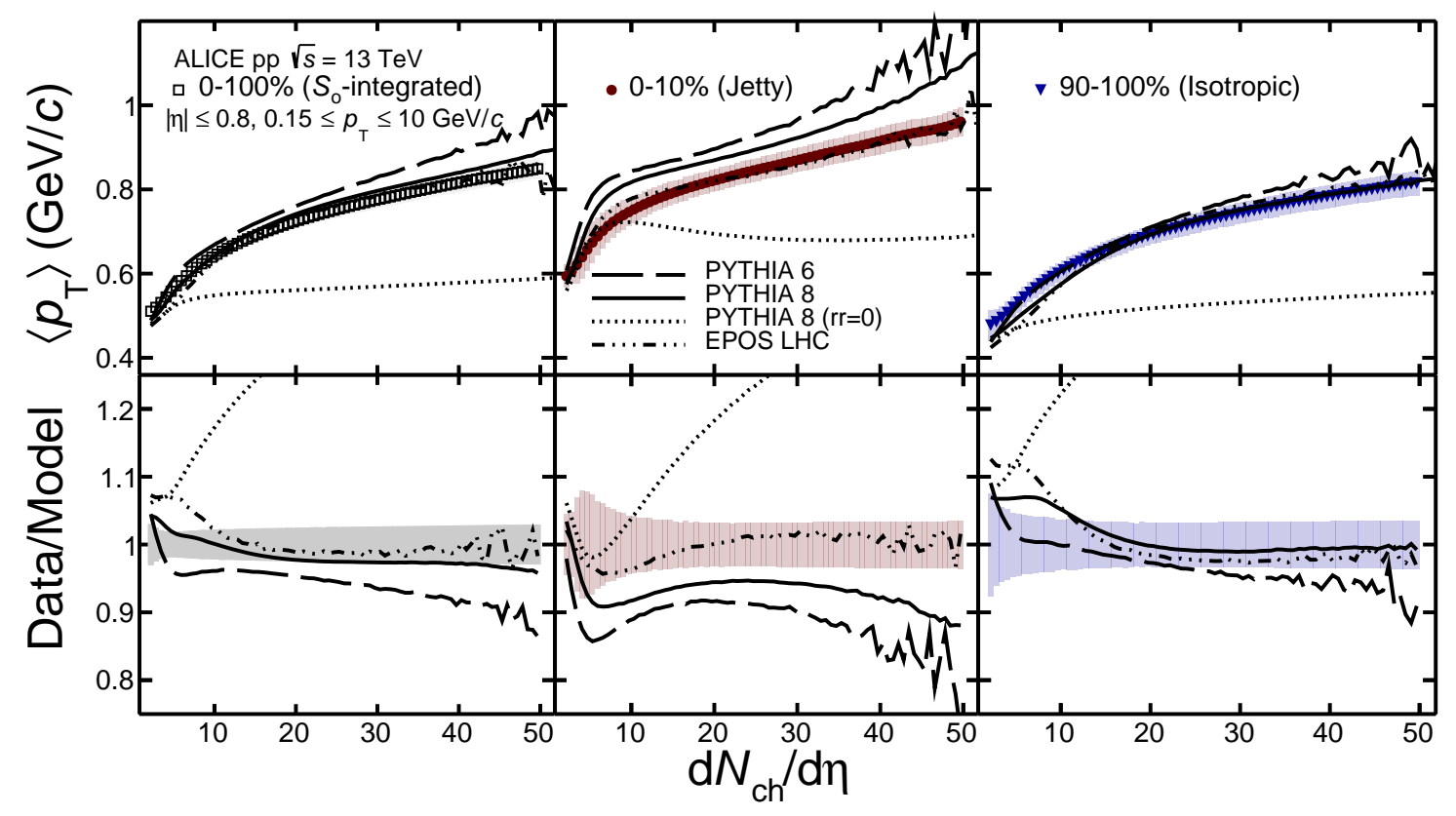

Figure 3: Average transverse momentum as a function of event multiplicity in pp collisions at $\sqrt{s}=$ $13 \mathrm{TeV}$ [13]. Results for the spherocity-integrated case (0-100\%), the most jet-like (0-10\%) and isotropic (90-100\%) events are compared with Monte Carlo predictions. Predictions of PYTHIA 8 with and without (null reconnection range, $\mathrm{rr}=0$ ) color reconnection, as well as PYTHIA 6 and EPOS LHC are displayed. Statistical uncertainties (error bars) are negligible compared to systematic uncertainties (shaded area around the data points). Data to model ratios are shown in the bottom panel. The color band around unity represents the systematic uncertainty.

of the strangeness enhancement in hadronic collisions and its relation to the QCD deconfinement phase transition are still open problems for models.

Given the strong correlation between multiplicity and particle production at high $p_{\mathrm{T}}$ observed in pp data [13], the identified $p_{\mathrm{T}}$ spectra at high $N_{\mathrm{ch}}$ have a non-negligible contribution from hard physics. In order to disentangle the soft and hard contribution, transverse spherocity was explored by ALICE. Spherocity is defined for a unit vector $\hat{\mathbf{n}}_{\mathbf{s}}$ which minimizes (min) the ratio:

$$
S_{0} \equiv \frac{\pi^{2}}{4} \min _{\hat{\mathbf{n}}_{\mathbf{s}}}\left(\frac{\sum_{i}\left|\vec{p}_{\mathrm{T}, i} \times \hat{\mathbf{n}}_{\mathbf{s}}\right|}{\sum_{i} p_{\mathrm{T}, i}}\right)^{2}
$$

where the sum runs over all primary charged particles. At least three particles are required within $|\eta|<0.8$ and $p_{\mathrm{T}}>0.15 \mathrm{GeV} / c$. It is worth mentioning some features of spherocity:

- Low values of spherocity $\left(S_{0} \rightarrow 0\right)$ correspond to jet-like event topologies.

- High values of spherocity $\left(S_{0} \rightarrow 1\right)$ correspond to event topologies where transverse momentum vectors are "isotropically" distributed.

ALICE measured the average transverse momentum as a function of multiplicity for different spherocity classes [13]. Figure 3 shows the average $p_{\mathrm{T}}$ as a function of $\mathrm{d} N_{\mathrm{ch}} / \mathrm{d} \eta$ for pp collisions at $\sqrt{s}=13 \mathrm{TeV}$. In accordance with measurements at lower energies [21], the $\left\langle p_{\mathrm{T}}\right\rangle$ increases with 
$\mathrm{d} N_{\mathrm{ch}} / \mathrm{d} \eta$. The minimum bias data (spherocity-integrated event class) are compared with analogous measurements for the most jet-like structure (0-10\%) and isotropic (90-100\%) event classes. Studying observables as a function of spherocity reveals interesting features. On one hand, for isotropic events the average $p_{\mathrm{T}}$ is systematically below the spherocity-integrated $\left\langle p_{\mathrm{T}}\right\rangle$ over the full multiplicity range; on the other hand, for jet-like events the $\left\langle p_{\mathrm{T}}\right\rangle$ is higher than that for spherocityintegrated events. Within uncertainties, PYTHIA 8 with color reconnection gives an adequate description of the spherocity-integrated event class. It is worth mentioning that color reconnection was originally introduced to explain the rise of $\left\langle p_{\mathrm{T}}\right\rangle$ with multiplicity [28], and it turned into a "QCD generator" of radial flow-like patterns [29]. However, PYTHIA 6 shows a steeper rise of $\left\langle p_{\mathrm{T}}\right\rangle$ with $\mathrm{d} N_{\mathrm{ch}} / \mathrm{d} \eta$ than that seen in data. The comparison of data with EPOS LHC is also shown. Clearly, the quantitative agreement is as good as that achieved by PYTHIA 8 . For the jettylike and isotropic events corresponding to $0-10 \%$ and $90-100 \%$ spherocity classes, respectively, Fig. 3 also shows comparisons between data and Monte Carlo generators (PYTHIA 6, PYTHIA 8 and EPOS LHC). In low-multiplicity events $\left(\mathrm{d} N_{\mathrm{ch}} / \mathrm{d} \eta<10\right)$, the deviations between data and PYTHIA 8 (without color reconnection) are smaller and larger respectively for the $0-10 \%$ and 90 $100 \%$ spherocity classes than those seen for the $0-100 \%$ spherocity class. The effect could be a consequence of the reduction of the color reconnection contribution in events containing jets surrounded by a small underlying event activity. For isotropic events the three models quantitatively describe the correlation. Even for PYTHIA 6, the size of the discrepancy which was pointed out for the spherocity-integrated event class is reduced. On the contrary, for jet-like events both PYTHIA 6 and 8 exhibit a larger disagreement with the data.

Last but not least, it is worth mentioning that another approach is under investigation. It is inspired on results from heavy-ion collisions, where particle production is studied in both the jet-like and bulk regions, separately. Namely, the proton-to-pion ratio as a function of $p_{\mathrm{T}}$ in the bulk region exhibits a bump structure at intermediate $p_{\mathrm{T}}$ [30]. No bump is observed in the jetlike region. Therefore, one can argue that the bump observed in central heavy-ion collisions is a medium-related effect. Similar results for the Lambda-to-kaon ratio were reported for $\mathrm{p}-\mathrm{Pb}$ collisions [31]. For pp collisions, an analogous separation implies the extension of the traditional underlying-event analysis [32-35].

\section{Multi-particle correlations}

The key question which can be addressed using multi-particle correlations is whether the ridge phenomenon reflects initial momentum correlations from gluon saturation, or a final-state hydrodynamic response to the initial transverse collision geometry. The multi-particle cumulants probe the event-by-event fluctuation of a single flow harmonic $v_{n}$, as well as the correlated fluctuations between the flow harmonics $v_{n}$ and $v_{m}$. As discussed above, the main challenge in small systems is how to disentangle the long-range ridge from "non-flow" correlations involving only few particles such as resonance decays or jets. For multi-particle cumulants, the non-flow contributions can be controlled by requiring correlations between particles from different subevents separated in $\eta$.

For example, for the recent results reported by ATLAS, the standard cumulant method consists on calculating the $k$-particle azimuthal correlations [36, 37], defined as:

$$
\left\langle\{2\}_{n}\right\rangle=\left\langle e^{i n\left(\phi_{1}-\phi_{2}\right)}\right\rangle,\left\langle\{3\}_{n}\right\rangle=\left\langle e^{i n\left(\phi_{1}+\phi_{2}-2 \phi_{3}\right)}\right\rangle,\left\langle\{4\}_{n, m}\right\rangle=\left\langle e^{i n\left(\phi_{1}-\phi_{2}\right)+i m\left(\phi_{3}-\phi_{4}\right)}\right\rangle
$$


Tracks within the entire acceptance of $|\eta|<\eta_{\max }=2.5$ are selected. The multi-particle asymmetric and symmetric cumulants are obtained as:

$$
\operatorname{ac}_{n}\{3\}=\left\langle\left\langle\{3\}_{n}\right\rangle\right\rangle, \mathrm{sc}_{n, m}\{4\}=\left\langle\left\langle\{4\}_{n, m}\right\rangle\right\rangle-\left\langle\left\langle\{2\}_{n}\right\rangle\right\rangle\left\langle\left\langle\{2\}_{m}\right\rangle\right\rangle,
$$

where $\langle\langle\rangle\rangle$ represents a weighted average of $\langle\{\mathrm{k}\}\rangle$ over an event sample with similar multiplicity. In the absence of non-flow correlations, $\operatorname{sc}_{n, m}\{4\}$ and $\operatorname{ac}_{n}\{3\}$ measure the correlation between $v_{n}$ and $v_{m}$ or between $v_{n}$ and $v_{2 n}$. Having said this, a technique to suppress further the non-flow correlations that typically involve a few particles within a localised region in $\eta$, has to be applied. Tracks are divided into several subevents, each covering a unique $\eta$ interval. The multi-particle correlations are then constructed by only correlating tracks between different subevents. In the two-subevent cumulant method, the tracks are divided into two subevents, labelled by $a$ and $b$, according to $-\eta_{\max }<\eta_{a}<0$ and $0<\eta_{b}<\eta_{\max }$. In the three-subevent cumulant method, tracks in each event are divided into three subevents $a, b$ and $c$, each covering one third of the $\eta$ range, $-\eta_{\max }<\eta_{a}<-\eta_{\max } / 3,\left|\eta_{b}\right| \leq \eta_{\max } / 3$ and $\eta_{\max } / 3<\eta_{c}<\eta_{\max }$. It has been shown that methods with three or more subevents are sufficient to reject now-flow correlation from jets.
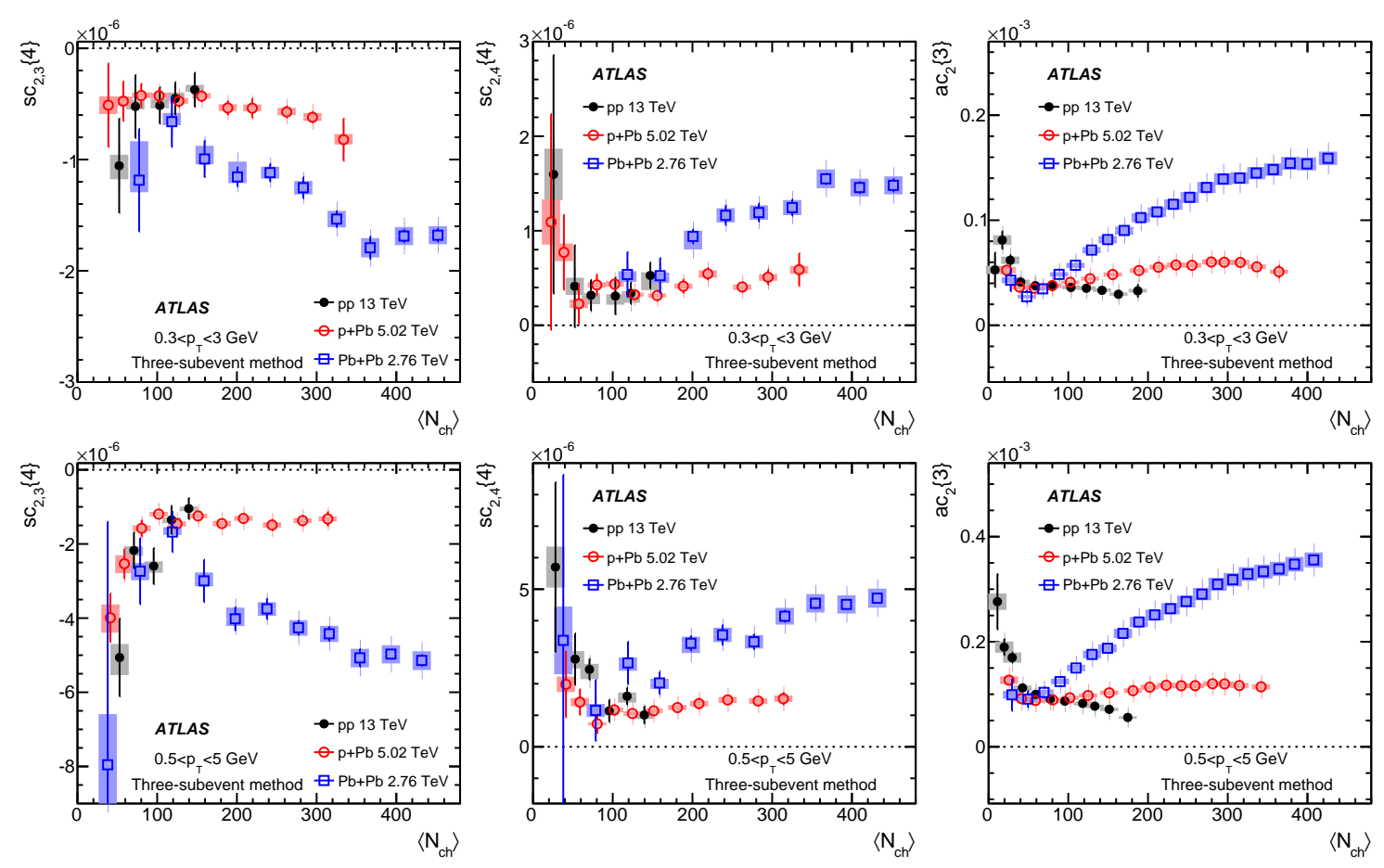

Figure 4: Multiplicity dependence of (left) $\mathrm{sc}_{2,3}\{4\}$, (middle) $\mathrm{sc}_{2,4}\{4\}$ and (right) ac $2\{3\}$ in (top) $0.3<$ $p_{\mathrm{T}}<3 \mathrm{GeV} / c$ and (bottom) $0.5<p_{\mathrm{T}}<5 \mathrm{GeV} / c$ obtained for pp collisions (solid circles), $\mathrm{p}-\mathrm{Pb}$ collisions (open circles) and low-multiplicity $\mathrm{Pb}-\mathrm{Pb}$ collisions (open squares) [38]. The error bars and shaded boxes represent the statistical and systematic uncertainties, respectively.

Figure 4 shows a comparison among results for $\mathrm{pp}, \mathrm{p}-\mathrm{Pb}$ and $\mathrm{Pb}-\mathrm{Pb}$ collisions at $\sqrt{s}_{\mathrm{NN}}=13$, 5.02 and $2.76 \mathrm{TeV}$, respectively [38]. Symmetric $\left(\mathrm{sc}_{2,3}\{4\}, \mathrm{sc}_{2,4}\{4\}\right)$ and asymmetric $\left(\mathrm{ac}_{2}\{3\}\right)$ cumulants, for $0.3<p_{\mathrm{T}}<3 \mathrm{GeV} / c$, are shown in the top panel. These results show a negative correlation between $v_{2}$ and $v_{3}$ and a positive correlation between $v_{2}$ and $v_{4}$. It is worth noticing that 
for the first time these patterns in small systems are observed, once non-flow effects are adequately suppressed. In the multiplicity range covered by the pp collisions, $\left\langle N_{\mathrm{ch}}\right\rangle<150$, the results for symmetric cumulants $\mathrm{sc}_{2,3}\{4\}$ and $\mathrm{sc}_{2,4}\{4\}$ are similar among the three systems. In the range $\left\langle N_{\mathrm{ch}}\right\rangle>150,\left|\mathrm{sc}_{2,3}\{4\}\right|$ and $\mathrm{sc}_{2,4}\{4\}$ are larger in $\mathrm{Pb}-\mathrm{Pb}$ than in $\mathrm{p}-\mathrm{Pb}$ collisions. The results for $\mathrm{ac}_{2}\{3\}$ are similar among the three systems at $\left\langle N_{\mathrm{ch}}\right\rangle<100$, but they deviate from each other at higher $\left\langle N_{\mathrm{ch}}\right\rangle$. The pp data are approximately constant or decrease slightly with $\left\langle N_{\mathrm{ch}}\right\rangle$, while the $\mathrm{p}-\mathrm{Pb}$ and $\mathrm{Pb}-\mathrm{Pb}$ data show significant increases as a function of $\left\langle N_{\mathrm{ch}}\right\rangle$. The bottom row shows the results for the higher $p_{\mathrm{T}}$ range of $0.5<p_{\mathrm{T}}<5 \mathrm{GeV} / c$, where similar trends are observed. Finally, for other collision systems such as $\mathrm{Xe}-\mathrm{Xe}$ and $\mathrm{Pb}-\mathrm{Pb}$ collisions at $\sqrt{s}_{\mathrm{NN}}=5.44$ and $5.02 \mathrm{TeV}$, respectively, a positive $\mathrm{sc}_{2,4}$ was observed [39]. While for $\mathrm{sc}_{2,4}$, results from ALICE, ATLAS and CMS qualitatively agree, the ALICE $\mathrm{sc}_{2,3}$ measurement goes from negative (anticorrelation between $v_{2}$ and $v_{3}$ ) to positive values at $N_{\mathrm{ch}} \sim 100$. Same tendency is found for $\mathrm{pp}$ and $\mathrm{p}-\mathrm{Pb}$ collisions. This different behavior is under investigation.

It has been shown that values of $\operatorname{sc}_{2,4}\{4\}$ and $\operatorname{ac}_{2}\{3\}$, which are both measures of correlations between $v_{2}$ and $v_{4}$, show significant differences between the standard method and the subevent methods. These differences seem to persist for $\left\langle N_{\mathrm{ch}}\right\rangle>200 \mathrm{in} \mathrm{p}-\mathrm{Pb}$ collisions and for $\left\langle N_{\mathrm{ch}}\right\rangle>150$ in $\mathrm{Pb}-\mathrm{Pb}$ collisions, which is not compatible with the predicted behaviour of non-flow correlations at large $\left\langle N_{\mathrm{ch}}\right\rangle$ [38]. The effect may arise from longitudinal flow decorrelations. The ATLAS and CMS collaborations measured decorrelation effects [40, 41], which are large for $v_{4}$ and strongly correlated with $v_{2}$, and therefore they are expected to reduce the $\operatorname{sc}_{2,4}\{4\}$ and $\mathrm{ac}_{2}\{3\}$ in the subevent method.

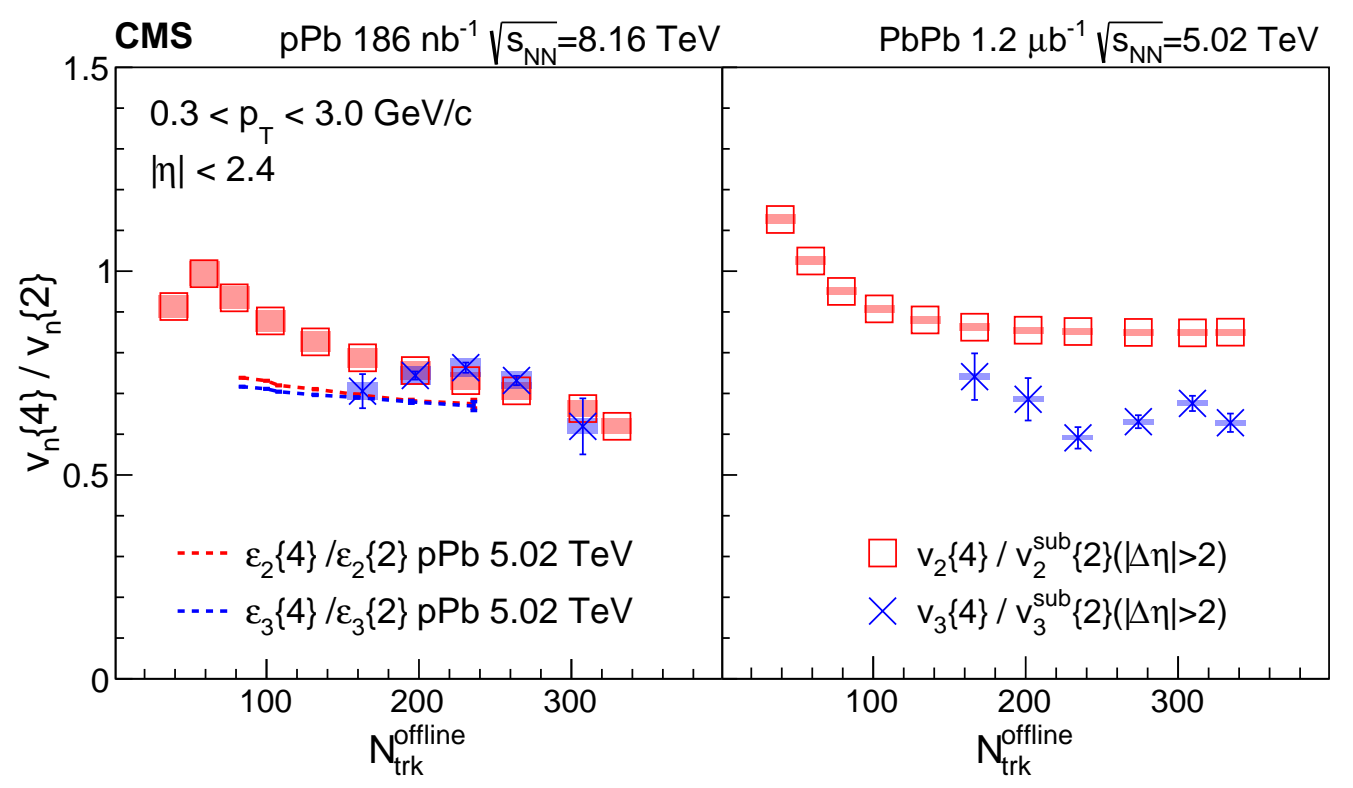

Figure 5: The ratios of four- and two-particle harmonics $\left(v_{2}\{4\} / v_{2} 2\right.$ and $\left.v_{3}\{4\} / v_{3}\{2\}\right)$ are shown as a function of event multiplicity for (left) $\mathrm{p}-\mathrm{Pb}$ collisions at $\sqrt{s_{\mathrm{NN}}}=8.16 \mathrm{TeV}$ and (right) $\mathrm{Pb}-\mathrm{Pb}$ collisions at $\sqrt{s_{\mathrm{NN}}}=5.02 \mathrm{TeV}$ [42]. Error bars and shaded boxes denote statistical and systematic uncertainties, respectively. The dashed curves show a hydrodynamics-motivated initial-state fluctuation calculation for $\mathrm{p}-\mathrm{Pb}$ collisions at $\sqrt{s_{\mathrm{NN}}}=5.02 \mathrm{TeV}$. 
From the hydrodynamic picture, the $v_{n}\{m\}$ values are expected to be proportional to initialstate eccentricities $\varepsilon_{n}\{m\}^{1}$, with $v_{n}\{m\}=k_{n} \varepsilon_{n}\{m\}$, where $k_{n}$ reflects the medium properties and does not depend on the order of the cumulant. Therefore, ratios of different cumulant $v_{n}$ values can directly probe properties of initial-state eccentricity. Using $\mathrm{p}-\mathrm{Pb}$ data at $\sqrt{s_{\mathrm{NN}}}=8.16 \mathrm{TeV}$, the CMS collaboration has improved the precision on the measurement of the $v_{2}$ harmonics. Moreover, for the first time the $v_{3}$ harmonic was determined using multi-particle correlations [42]. This allows the ratios $v_{2}\{4\} / v_{2}\{2\}$ and $v_{3}\{4\} / v_{3}\{2\}$ to be obtained in order to probe the properties of initial-state eccentricity. Figure 5 shows the ratios $v_{2}\{4\} / v_{2}\{2\}$ and $v_{3}\{4\} / v_{3}\{2\}$ for both $\mathrm{p}-\mathrm{Pb}$ and $\mathrm{Pb}-\mathrm{Pb}$ collisions. For $\mathrm{p}-\mathrm{Pb}$ collisions, the ratios for $v_{2}$ and $v_{3}$ are similar within uncertainties, which is consistent with having both the second- and third-order harmonics arising from the same initialstate fluctuation mechanism. Comparing the $\mathrm{p}-\mathrm{Pb}$ and $\mathrm{Pb}-\mathrm{Pb}$ data, the $v_{3}$ ratios are comparable for both systems, while the $v_{2}$ ratios are higher in $\mathrm{Pb}-\mathrm{Pb}$ than in $\mathrm{p}-\mathrm{Pb}$ for higher multiplicity values, again reflecting the larger geometric contribution for the larger collision system. The figure also shows that a hydrodynamics-motivated initial-state fluctuation calculation of eccentricities for $\mathrm{p}$ $\mathrm{Pb}$ collisions at $\sqrt{s_{\mathrm{NN}}}=5.02 \mathrm{TeV}$, qualitatively describes the data.

\section{Summary}

In this work, selected results on particle correlations and transverse momentum spectra in small systems have been presented. From the experimental side, new techniques were developed in order to improve the removal of trivial correlations from flow measurements. For example, using the sub-event method a non zero $v_{2}$ is observed for $\mathrm{pp}$ and $\mathrm{p}-\mathrm{Pb}$ collisions in accordance with hydrodynamic predictions. Moreover, the anti-correlation between $v_{2}$ and $v_{3}$ (as expected from hydrodynamics) in small systems has been reported by ATLAS and CMS. However, ALICE data exhibit a different behavior for low multiplicity events, this difference needs to be fully understood. Regarding transverse momentum spectra as a function of multiplicity, radial flow-like behavior is observed in data, and now the role of autocorrelations is under investigation using an event selection based on transverse spherocity. New measurements of strange and multistrange particles as a function of multiplicity have been performed. The MC event generators can not fully describe the data.

We are still far from a complete understanding of the new phenomena discovered in small systems. More work needs to be done both from the theory and experimental sides, in order to find a unified description of the system size dependence of particle production.

\section{Acknowledgments}

The presenter has been supported by PAPIIT-UNAM under the Grant No. IN102118.

\footnotetext{
${ }^{1}$ Initial-state eccentricities are characterized by the cumulants of the event-by-event distributions of their Fourier harmonics coefficients.
} 


\section{References}

[1] W. Busza, K. Rajagopal, and W. van der Schee, "Heavy Ion Collisions: The Big Picture, and the Big Questions," Ann. Rev. Nucl. Part. Sci. 68 (2018) 339-376, arXiv: 1802.04801 [hep-ph] .

[2] P. Romatschke and U. Romatschke, Relativistic Fluid Dynamics In and Out of Equilibrium. Cambridge Monographs on Mathematical Physics. Cambridge University Press, 2019. arXiv:1712.05815 [nucl-th].

[3] G.-Y. Qin and X.-N. Wang, "Jet quenching in high-energy heavy-ion collisions," Int. J. Mod. Phys. E24 no. 11, (2015) 1530014, arXiv:1511.00790 [hep-ph] . [,309(2016)].

[4] CMS Collaboration, V. Khachatryan et al., "Observation of Long-Range Near-Side Angular Correlations in Proton-Proton Collisions at the LHC," JHEP 09 (2010) 091, arXiv:1009.4122 [hep-ex].

[5] CMS Collaboration, V. Khachatryan et al., "Evidence for collectivity in pp collisions at the LHC," Phys. Lett. B765 (2017) 193-220, arXiv:1606.06198 [nucl-ex] .

[6] ALICE Collaboration, B. Abelev et al., "Long-range angular correlations on the near and away side in $p-\mathrm{Pb}$ collisions at $\sqrt{s_{N N}}=5.02 \mathrm{TeV}$," Phys. Lett. B719 (2013) 29-41, arXiv:1212.2001 [nucl-ex].

[7] LHCb Collaboration, R. Aaij et al., "Measurements of long-range near-side angular correlations in $\sqrt{s_{\mathrm{NN}}}=5 \mathrm{TeV}$ proton-lead collisions in the forward region," Phys. Lett. B762 (2016) 473-483, arXiv:1512.00439 [nucl-ex].

[8] P. Bozek, "Collective flow in p-Pb and d-Pd collisions at TeV energies," Phys. Rev. C85 (2012) 014911, arXiv:1112.0915 [hep-ph] .

[9] P. M. Chesler, "Colliding shock waves and hydrodynamics in small systems," Phys. Rev. Lett. 115 no. 24, (2015) 241602, arXiv:1506.02209 [hep-th].

[10] S. Schlichting and P. Tribedy, "Collectivity in Small Collision Systems: An Initial-State Perspective,” Adv. High Energy Phys. 2016 (2016) 8460349, arXiv:1611. 00329 [hep-ph] .

[11] M. Strickland, "Small system studies: A theory overview," Nucl. Phys. A982 (2019) 92-98, arXiv:1807.07191 [nucl-th].

[12] Z.-W. Lin, C. M. Ko, B.-A. Li, B. Zhang, and S. Pal, "A Multi-phase transport model for relativistic heavy ion collisions," Phys. Rev. C72 (2005) 064901, arXiv:nucl-th/0411110 [nucl-th].

[13] ALICE Collaboration, S. Acharya et al., "Charged-particle production as a function of multiplicity and transverse spherocity in pp collisions at $\sqrt{s}=5.02$ and $13 \mathrm{TeV}$," arXiv:1905.07208 [nucl-ex]. 
[14] J. L. Nagle and W. A. Zajc, "Small System Collectivity in Relativistic Hadronic and Nuclear Collisions," Ann. Rev. Nucl. Part. Sci. 68 (2018) 211-235, arXiv:1801.03477 [nucl-ex].

[15] ALICE Collaboration, S. Acharya et al., "Multiplicity dependence of light-flavor hadron production in pp collisions at $\sqrt{s}=7 \mathrm{TeV}$," Phys. Rev. C99 no. 2, (2019) 024906, arXiv:1807.11321 [nucl-ex].

[16] ALICE Collaboration, J. Adam et al., "Multiplicity dependence of charged pion, kaon, and (anti)proton production at large transverse momentum in $\mathrm{p}-\mathrm{Pb}$ collisions at $\sqrt{s_{\mathrm{NN}}}=5.02$ TeV," Phys. Lett. B760 (2016) 720-735, arXiv:1601.03658 [nucl-ex] .

[17] ALICE Collaboration, B. B. Abelev et al., "Multiplicity Dependence of Pion, Kaon, Proton and Lambda Production in p-Pb Collisions at $\sqrt{s_{N N}}=5.02 \mathrm{TeV}$," Phys. Lett. B728 (2014) 25-38, arXiv:1307.6796 [nucl-ex].

[18] ALICE Collaboration, J. Adam et al., "Enhanced production of multi-strange hadrons in high-multiplicity proton-proton collisions," Nature Phys. 13 (2017) 535-539, arXiv:1606.07424 [nucl-ex].

[19] J. Cleymans, I. Kraus, H. Oeschler, K. Redlich, and S. Wheaton, "Statistical model predictions for particle ratios at $\mathrm{s}(\mathrm{NN}) * *(1 / 2)=5.5-\mathrm{TeV}$," Phys. Rev. C74 (2006) 034903, arXiv:hep-ph/0604237 [hep-ph].

[20] A. Andronic, P. Braun-Munzinger, and J. Stachel, "Thermal hadron production in relativistic nuclear collisions: The Hadron mass spectrum, the horn, and the QCD phase transition," Phys. Lett. B673 (2009) 142-145, arXiv:0812.1186 [nucl-th]. [Erratum: Phys. Lett.B678,516(2009)].

[21] S. Wheaton and J. Cleymans, "THERMUS: A Thermal model package for ROOT," Comput. Phys. Commun. 180 (2009) 84-106, arXiv: hep-ph/ 0407174 [hep-ph].

[22] V. Vislavicius and A. Kalweit, "Multiplicity dependence of light flavour hadron production at LHC energies in the strangeness canonical suppression picture," arXiv:1610.03001 [nucl-ex].

[23] ALICE Collaboration, S. Acharya et al., "Multiplicity dependence of (multi-)strange hadron production in proton-proton collisions at $\sqrt{s}=13 \mathrm{TeV}$," arXiv: 1908.01861 [nucl-ex].

[24] T. Sjöstrand, S. Ask, J. R. Christiansen, R. Corke, N. Desai, P. Ilten, S. Mrenna, S. Prestel, C. O. Rasmussen, and P. Z. Skands, "An Introduction to PYTHIA 8.2," Comput. Phys. Commun. 191 (2015) 159-177, arXiv:1410.3012 [hep-ph].

[25] P. Skands, S. Carrazza, and J. Rojo, “Tuning PYTHIA 8.1: the Monash 2013 Tune," Eur. Phys. J. C74 no. 8, (2014) 3024, arXiv:1404.5630 [hep-ph]. 
[26] C. Flensburg, G. Gustafson, and L. Lonnblad, "Inclusive and Exclusive Observables from Dipoles in High Energy Collisions," JHEP 08 (2011) 103, arXiv: 1103.4321 [hep-ph] .

[27] K. Werner, "Core-corona separation in ultra-relativistic heavy ion collisions," Phys. Rev. Lett. 98 (2007) 152301, arXiv:0704.1270 [nucl-th].

[28] T. Sjöstrand and M. van Zijl, "A Multiple Interaction Model for the Event Structure in Hadron Collisions," Phys. Rev. D36 (1987) 2019.

[29] A. Ortiz, P. Christiansen, E. Cuautle Flores, I. Maldonado Cervantes, and G. Paić, "Color Reconnection and Flowlike Patterns in pp Collisions," Phys. Rev. Lett. 111 no. 4, (2013) 042001, arXiv:1303.6326 [hep-ph].

[30] ALICE Collaboration, M. Veldhoen, " $p / \pi$ Ratio in Di-Hadron Correlations," Nucl. Phys. A910-911 (2013) 306-309, arXiv:1207.7195 [nucl-ex].

[31] T. O. H. Richert, $\Lambda / \mathrm{K}_{\mathrm{s}}^{0}$ associated with a jet in central Pb-Pb collisions at $\sqrt{s_{\mathrm{NN}}}=2.76 \mathrm{TeV}$ measured with the ALICE detector. PhD thesis, Lund U., 2016-05-16.

[32] CDF Collaboration, R. D. Field, "The Underlying event in hard scattering processes," eConf C010630 (2001) P501, arXiv: hep-ph/0201192 [hep-ph] .

[33] T. Martin, P. Skands, and S. Farrington, "Probing Collective Effects in Hadronisation with the Extremes of the Underlying Event," Eur. Phys. J. C76 no. 5, (2016) 299, arXiv:1603.05298 [hep-ph].

[34] A. Ortiz and L. Valencia Palomo, "Universality of the underlying event in pp collisions," Phys. Rev. D96 no. 11, (2017) 114019, arXiv:1710.04741 [hep-ex] .

[35] A. Ortiz and L. Valencia Palomo, "Probing color reconnection with underlying event observables at the LHC energies," Phys. Rev. D99 no. 3, (2019) 034027, arXiv:1809.01744 [hep-ex].

[36] N. Borghini, P. M. Dinh, and J.-Y. Ollitrault, "Flow analysis from multiparticle azimuthal correlations," Phys. Rev. C64 (2001) 054901, arXiv: nucl-th/0105040 [nucl-th].

[37] A. Bilandzic, R. Snellings, and S. Voloshin, "Flow analysis with cumulants: Direct calculations," Phys. Rev. C83 (2011) 044913, arXiv:1010.0233 [nucl-ex] .

[38] ATLAS Collaboration, M. Aaboud et al., "Correlated long-range mixed-harmonic fluctuations measured in $\mathrm{pp}, \mathrm{p}-\mathrm{Pb}$ and low-multiplicity $\mathrm{Pb}-\mathrm{Pb}$ collisions with the ATLAS detector," Phys. Lett. B789 (2019) 444-471, arXiv:1807.02012 [nucl-ex].

[39] ALICE Collaboration, S. Acharya et al., "Investigations of anisotropic flow using multi-particle azimuthal correlations in pp, $\mathrm{p}-\mathrm{Pb}, \mathrm{Xe}-\mathrm{Xe}$, and $\mathrm{Pb}-\mathrm{Pb}$ collisions at the LHC," arXiv:1903.01790 [nucl-ex]. 
[40] ATLAS Collaboration, M. Aaboud et al., "Measurement of longitudinal flow decorrelations in $\mathrm{Pb}+\mathrm{Pb}$ collisions at $\sqrt{s_{\mathrm{NN}}}=2.76$ and $5.02 \mathrm{TeV}$ with the ATLAS detector," Eur. Phys. J. C78 no. 2, (2018) 142, arXiv:1709.02301 [nucl-ex].

[41] CMS Collaboration, V. Khachatryan et al., "Evidence for transverse momentum and pseudorapidity dependent event plane fluctuations in $\mathrm{Pb}-\mathrm{Pb}$ and $\mathrm{p}-\mathrm{Pb}$ collisions," Phys. Rev. C92 no. 3, (2015) 034911, arXiv:1503.01692 [nucl-ex].

[42] CMS Collaboration, A. M. Sirunyan et al., "Multiparticle correlation studies in $\mathrm{pPb}$ collisions at $\sqrt{s_{\mathrm{NN}}}=8.16 \mathrm{TeV}$," arXiv:1904.11519 [hep-ex] . 